\title{
The answer is not blowin' in the wind, or is it?
}

\author{
Santanu Bandyopadhyay
}

Published online: 5 February 2014

(c) Springer-Verlag Berlin Heidelberg 2014

Energy is one of the most important prime movers for our civilization. It is indispensable for continued development and growth of our civilization. Adequate 'consumption' of energy is one of most important prerequisites to address various aspects of human developments. Thermodynamics teaches us that 'the energy can neither be created nor destroyed; it can only be transformed from one form to another'. During this transformation process, we degrade its quality. In other words, we transform high quality energy to low quality energy-colloquially termed as energy 'consumption'. Starting point of the energy transformation process is the primary energy, the form of energy that is directly available in nature (e.g. solar radiation, biomass, fossil fuels, etc.). In most cases, primary energy is transformed into a desirable and suitable form- secondary energy (e.g. electricity, gasoline, jet fuel, etc.). This is finally transformed to various energy services or end-use.

During pre- and early industrial era, biomass and some locally available renewable energy sources such as solar, water, tide, wind and muscle power of human and animal used to form the primary energy mix. The muscle powers of both human and animal were used to drive simple machines since pre-historic times and even used today in many parts of the world. We still use the term 'horsepower', to define capacity of many modern machines, as a historical hangover to remind us about the role of muscle power in our development.

Discovery and utilization of fossil fuel (i.e. coal, oil and natural gas) played a catalytic role during industrialization era, and to date, fossil fuel dominates the primary energy

S. Bandyopadhyay $(\square)$

Department of Energy Science and Engineering, Indian Institute

of Technology Bombay, Powai 400076, Mumbai, India

e-mail: santanub@iitb.ac.in mix. Discovery of these reliable and seemingly 'inexhaustible' energy sources along with the quest for powerful and sophisticated machineries led to the industrial revolution. It is interesting to note that during early industrial period, sun was also viewed as a possible energy source and in the year 1767, Swiss scientist Horace de Saussure developed the first solar thermal collector. However, such developments slowly faded away due availability of accessible, dependable, inexpensive and transportable fossil fuels in large quantities.

During the last few decades, a fundamental change in primary energy mix started creeping in. We started realizing that seemingly 'inexhaustible' fossil fuel sources are actually finite. The linkage between various environmental problems as well as effect of carbon dioxide in global climate change, and utilization of fossil fuel has been established beyond doubt. Various non-conventional energy sources (such as wind, photovoltaic, hydro, etc.) along with nuclear energy started contributing to the overall primary energy mix to de-carbonize the overall energy systems. Thus, the very fundamental growth model got questioned.

The most important question is related to the exponential growth model vis-à-vis sustainable development of the entire human civilization. A sustainable energy system should be able to provide energy services to the present generation while ensuring that similar levels of energy services can be provided for future generations without any compromise. It is important to note that a sustainable energy system by itself cannot ensure sustainable development of the entire society. But, this is one of the very important steps towards developing an overall sustainable society. The major solutions towards developing a sustainable energy system are improving energy efficiency, improved conversion efficiency, and appropriate utilization of the renewable energy sources. Nuclear energy can help 
in de-carbonizing the present energy system. However, increasing public resistance to nuclear energy, especially after the 2011 Fukushima disaster, started challenging future prospect of nuclear energy. Lower operating cost, reduced environmental emissions, and modularity in design and thereby distributed applications, along with ease of expansion are the major advantages of renewable energy systems. Low energy density, high initial capital investment and relatively poor reliability and availability are the disadvantages associated with them. With rapidly increasing energy demands in the developing countries, it may be impractical to switch to renewable energy-based solutions in immediate future. Carbon capture and storage can provide an intermediate solution to this issue.

Another important aspect of sustainable development is equitability. Energy consumption is not distributed equally and equitably. Per capita energy consumption is extremely high in North America and extremely low in South Asia and sub-Saharan Africa. It is a serious threat to the overall development of human civilization leading to the very important question on how affordable energy can be easily accessed by all to fulfill basic human developments.

We have reached a crossroad of energy utilization. We need to answer questions on development of our civilization, accessibility of energy, improved conversion of existing energy systems and energy services, environmental issues for our very existence, development of cleaner energy sources, development of various policies for our holistic and sustainable growth, etc. These important and relevant issues cannot be addressed in isolation. Pages of Clean Energy and Environmental Policy are dedicated to answering the above questions. 